\title{
Les alliances éducatives pour lutter contre le décrochage scolaire, Jean-Luc Gilles, Pierre Potvin, Chantal Tièche Christinat
}

Peter Lang éditeur - Éditions scientifiques internationales Berne 2012

\section{Anne-Marie Bardi}

\section{OpenEdition}

Journals

Édition électronique

URL : https://journals.openedition.org/ries/3096

DOI : 10.4000/ries.3096

ISSN : 2261-4265

Éditeur

France Education international

Édition imprimée

Date de publication : 1 avril 2013

Pagination : 25-27

ISBN : 978-2-85420-599-2

ISSN : $1254-4590$

\section{Référence électronique}

Anne-Marie Bardi, «Les alliances éducatives pour lutter contre le décrochage scolaire, Jean-Luc Gilles, Pierre Potvin, Chantal Tièche Christinat », Revue internationale d'éducation de Sèvres [En ligne], 62 I avril 2013, mis en ligne le 01 avril 2013, consulté le 05 juillet 2021. URL : http://journals.openedition.org/ ries/3096 ; DOI : https://doi.org/10.4000/ries.3096

Ce document a été généré automatiquement le 5 juillet 2021

(c) Tous droits réservés 


\title{
Les alliances éducatives pour lutter contre le décrochage scolaire, Jean-Luc Gilles, Pierre Potvin, Chantal Tièche Christinat
}

Peter Lang éditeur - Éditions scientifiques internationales Berne 2012

\author{
Anne-Marie Bardi
}

\section{RÉFÉRENCE}

Les alliances éducatives pour lutter contre le décrochage scolaire, Jean-Luc Gilles, Pierre

Potvin, Chantal Tièche Christinat. Peter Lang éditeur - Éditions scientifiques

internationales Berne 2012

1 En 2010, lors de son $16^{\mathrm{e}}$ congrès, l'Association mondiale des sciences de l'éducation (AMSE) a choisi pour thème : « les alliances éducatives pour lutter contre le décrochage scolaire». L'importance quantitative du décrochage scolaire et la gravité de ses conséquences individuelles, familiales, économiques et sociales ont suscité, depuis son apparition dans de nombreux pays, au cours des années quatre-vingts, des recherches touchant à plusieurs domaines : sociologie, psychologie, sciences de l'éducation. Toutes les études pointent la multi-factorialité du phénomène.

2 Les nombreuses causes $d u$ décrochage scolaire sont classées en déterminants « internes » et « externes » au système scolaire. Au titre des déterminants internes, on distingue des causes relevant de l'organisation et des structures (redoublement, orientation précoce, filières de relégation, etc.) et d'autres qui sont liées à la pédagogie (relations individuelles, gestion du temps de classe, effet Pygmalion, etc.). Les déterminants externes se divisent également en causes liées à la famille et aux relations sociales (pauvreté, monoparentalité, niveau d'étude, types d'amis et de «pairs ») et en 
causes individuelles (faible compétence en lecture, faible estime de soi, tendance dépressive, consommation de psychotropes, etc.).

3 Agir sur ces divers facteurs implique des interventions multiples, d'où la notion «d'alliances » retenue pour le colloque, notion plus forte que celle de coordination ou de complémentarité : l'alliance suppose des buts partagés ainsi que des priorités et des formes d'action définies en commun. Au niveau « micro », l'alliance concerne l'école, l'élève et sa famille, au niveau "méso », des psychologues, des éducateurs, des personnels de santé, des structures «relais»; enfin, au niveau "macro», elle rassemble les régions, les politiques, les syndicats, les entreprises.

4 Les diverses communications présentées lors du colloque, éclairées par les débats, puis réécrites et revues par des pairs, sont rassemblées dans un ouvrage qui fait un état de la question, sous ses aspects théoriques tout d'abord, puis avec des points de vue issus de divers pays. Une enquête " qualité » a permis d'ajouter en conclusion des orientations pour des recherches futures.

5 La première partie de l'ouvrage se centre sur la diversité des situations de décrochage scolaire et revient sur quelques idées reçues: C. Blaya dénonce l'amalgame entre absentéisme, décrochage, violence et délinquance. Les absentéistes restent chez eux pour $60 \%$ d'entre eux ; s'allier avec les familles vaut donc mieux que les stigmatiser. Au Luxembourg, à l'interface entre le jeune, sa famille et le marché de l'emploi, une structure ad hoc réalise une autre forme d'alliance : elle travaille sur les représentations de chacun et gère avec souplesse les transitions parfois chaotiques des jeunes vers l'entreprise. Enfin, une recherche portant sur les liens entre problèmes de comportement et décrochage scolaire, appuyée sur le suivi de près de 800 élèves durant leurs études secondaires, identifie les facteurs qui font basculer les problèmes de comportement vers le décrochage ou permettent au contraire de l'éviter.

6 La seconde partie rassemble des exemples d'alliances éducatives à différents niveaux. La gestion de classe, reconnue comme un acte professionnel complexe, est une forme d'alliance entre l'enseignant et l'élève : valeurs partagées et relations socio-affectives mais aussi création d'un climat sécurisant et d'un contexte favorable aux apprentissages diminuent le décrochage. Une étude menée en Suisse dans le canton de Vaud porte sur les réponses pédagogiques ou structurelles paradoxales préconisées par les enseignants : alors qu'ils privilégient les causes familiales parmi les 44 facteurs de décrochage proposés, ils suggèrent des solutions externes - structures (passerelles, relais) ou intervenants spécialisés - et passent sous silence les aspects pédagogiques. Plusieurs exemples québécois montrent l'engagement déjà ancien dans la lutte contre ce fléau et l'importance des recherches et des évaluations déjà effectuées. Ils analysent différentes approches, scolaires et communautaires ; sont ainsi décrites la mobilisation réussie de toute une collectivité territoriale (Saguenay-lac Saint-Jean) au plus haut niveau politique et économique, l'initiative gouvernementale « Stratégie d'intervention agir autrement » et les facteurs favorisant l'innovation et la diffusion des pratiques attestées.

7 La troisième partie présente des situations très ancrées dans leur contexte national. Le statut de l'école en France, sa structure, les attentes des familles et les formes d'enseignement génèreraient de l'échec scolaire, véritable «maladie nosocomiale de l'école» (Longhi); il faudrait donc «déscolariser la forme scolaire» pour traiter le problème... En Belgique, la multiplicité des réponses institutionnelles et des types d'intervenants occasionnait de la confusion ; une action systématique a permis de faire 
se rencontrer et se comprendre les acteurs et de créer des outils de coordination locale efficaces. La Bolivie cherche ses solutions dans une forte délégation des questions scolaires aux nombreuses "nations», entités linguistiques et culturelles qui ne partagent pas la même langue, la même religion ni la même vision du monde. La Suède semble avoir perdu aujourd'hui son modèle d'intégration et de réussite pour tous, en ayant appliqué à l'éducation des critères économiques de performance, de rationalisation et d'évaluation. Au Québec, les écueils qui freinent une nécessaire coopération entre chercheurs et praticiens sont explicités, ainsi que les facteurs de nature à faciliter leur coopération.

8 Cet ouvrage a le mérite de rappeler les acquis des recherches récentes sur le décrochage scolaire (ses diverses formes et ses causes multiples), d'apporter des analyses nouvelles sur des tensions qui restent à élucider, de fournir des exemples concrets d'alliances éducatives, fructueuses ou non, et d'ouvrir sur de futurs projets de recherche, le tout avec des contributions dépassant le strict cadre de la francophonie. Un petit regret toutefois : autocentré sur la recherche - ses méthodes, ses résultats, ses développements escomptés - cet ouvrage cherche trop peu l'alliance avec un lecteur extérieur : praticiens de l'éducation, responsables de structures scolaires, collectivités territoriales, hommes politiques se voient informés mais peu impliqués par cette lecture. Un petit paradoxe pour un ouvrage qui traite, justement, des «alliances éducatives »!

\section{AUTEURS}

\section{ANNE-MARIE BARDI}

Inspectrice générale honoraire de l'éducation nationale. Ancienne élève de l'École normale supérieure de Fontenay-aux Roses, agrégée de mathématiques, elle a été professeur puis inspectrice d'académie-inspectrice pédagogique régionale de mathématiques. Dès le début de l'informatique scolaire dans les années 1970, puis dans ses différentes fonctions, elle s'est impliquée dans le développement de l'usage des technologies de l'information et de la communication dans l'enseignement en France et à l'étranger. Elle est membre du comité de rédaction de la Revue internationale d'éducation de Sèvres. 